\title{
VIABILIDADE DA IMPLANTAÇÃO DE UM CONTRATO DE COMERCIALIZAÇÃO FUTURA DA MADEIRA DE REFLORESTAMENTO NO BRASIL $^{1}$
}

\author{
Naisy Silva Soares ${ }^{2}$, Fabiano Luiz da Silva², Márcio Lopes da Silva ${ }^{3}$, Aziz Galvão da Silva Júnior ${ }^{4}$ e \\ Viviani Silva Lírio ${ }^{4}$
}

\begin{abstract}
RESUMO - Este estudo analisou a viabilidade de implantação de um contrato futuro da madeira de Eucalyptus spp. para celulose. Para isso, tomou-se como referência a abordagem micro do modelo de Pennings e Leuthold (1999). Os dados foram coletados por meio de questionários direcionados às empresas de celulose. Os resultados indicaram que os gestores principais das empresas possuem características de indivíduos propensos a negociar em bolsas, como elevado nível de escolaridade e idade abaixo de 60 anos. Além disso, tais resultadosevidenciaram uma provável demanda por contratos futuros de madeira pelas empresas e que estas possuem condições financeiras suficientes para operar em bolsas, estão expostas e são avessas ao risco e que os funcionários de quase todas elas recebem treinamento nas áreas de economia e administração. Portanto, concluiu-se que existe grande potencial para a implantação de um contrato futuro desse tipo de madeira no Brasil.
\end{abstract}

Palavras-chave: Madeira, celulose e contrato futuro.

\section{VIABILITY OF IMPLANTATION OF A FUTURES CONTRACT OF WOOD REFORESTATION IN BRAZIL}

\begin{abstract}
This study analyzed the viability of implantation of futures contract of reforestation wood in Brazil, with focus on the market of Eucalyptus pulpwood production. For this purpose, the micro analysis model of Pennings and Leuthold (1999) was taken as reference. Data were collected through questionnaires addressed to the cellulose companies. The results indicated that the characteristics of managers and companies were shown favorable to the development of this type contract, since all the managers present individual characteristics prone to negotiate in exchange, as high education level and age below 60 years. Besides, the results showed a probable demand for future wood contracts in Brazil by the companies, and that they have enough financial conditions to operate in exchanges, are exposed to risk and are risk averse, and that employees of almost all the companies receive training in the economic and administration areas. It was therefore concluded that there is potential for the implantation of a futures contract of pulpwood in Brazil.
\end{abstract}

Keywords: Wood, wood pulp and futures contract.

\section{INTRODUÇÃO}

O investimento em reflorestamento no Brasil mostrase promissor, pois o país apresenta condições físicas e naturais para o desenvolvimento da atividade, e a produção da indústria brasileira, que tem como base madeira de reflorestamento, está crescendo devido ao aumento de sua exportação e ao surgimento de novos produtos que utilizam basicamente essa matéria-prima. As empresas florestais, que antes eram auto-suficientes em matéria-prima florestal, agora estão tendo que recorrer ao mercado e introduzir programas de fomento florestal para garantir o seu suprimento. Entretanto, os projetos

\footnotetext{
${ }^{1}$ Recebido em 26.10.2006 e aceito para publicação em 15.12.2006.

${ }^{2}$ ProgramadePós-GraduaçãoemCiênciaFlorestaldaUniversidadeFederal deViçosa.E-mail:<naisysilva@yahoo.com.br;fabianocoop@yahoo.com.br>.

${ }^{3}$ Departamento de Engenharia Florestal da Universidade Federal de Viçosa. E-mail: <marlosil@ ufv.br>.

${ }^{4}$ Departamento de Economia Rural da Universidade Federal de Viçosa. E-mail:<azziz@ufv.br; vslirio@ufv.br>.
} 
florestais caracterizam-se pelo elevado investimento inicial, longo tempo de maturação, retorno no longo prazo e riscos de ocorrência de incêndios, de ataques de pragas e doenças e de variações nos preços etc. Devido ao longo tempo de maturação, o retorno do investimento só ocorre no longo prazo. Além disso, o produtor florestal tem que tomar decisão de investimento, baseando-se no preço corrente do produto, uma vez que o preço futuro é desconhecido.

Acredita-se que um mercado dos contratos futuros da madeira no Brasil poderia oferecer aos produtores um referencial de preços futuros, auxiliando-os na tomadas de decisão e protegendo-os contra variações nos preços do produto, contribuindo, assim, para o aumento na área reflorestada no país.

Como um contrato futuro oferece benefícios para produtores e consumidores, alguns autores estudaram o potencial ou a viabilidade de se implantar um contrato futuro de determinado produto no Brasil. Dentre esses benefícios, citam-se Siqueira (2003) e Santos (2001), que alisaram, respectivamente, o potencial de implantação de um contrato futuro de leite e suínos no país.

Nesse contexto, o objetivo geral deste trabalho consiste em estudar o potencial de desenvolvimento de um contrato futuro de comercialização da madeira de Eucalyptus spp para celulose no Brasil, bem como identificar os fatores que podem contribuir para o sucesso e fracasso deste contrato.

\section{MATERIAL E MÉTODOS}

Para estudar o potencial de desenvolvimento de um contrato futuro de comercialização da madeira no Brasil e os fatores determinantes de seu sucesso ou fracasso, utilizou-se a abordagem micro do modelo de Pennings e Leuthold (1999). Tal abordagem focaliza os fatores que influenciam a tomada de decisão sobre optar ou não pelo uso de contratos futuros. Com essa abordagem, é possível identificar características das empresas e dos respectivos gerentes e, assim, relacionar com a decisão de usar ou não contratos futuros. Para tanto, são analisados fatores como aversão ao risco, oportunidade de crescimento, nível de riqueza, custos, nível de experiência do gerente da empresa, grau de escolaridade, idade, tamanho do empreendimento e utilização de mecanismos de gerenciamento de risco, entre outros.

R. Árvore, Viçosa-MG, v.31, n.2, p.307-314, 2007
Segundo Pennings e Leuthold (1999), a abordagem micro responde à seguinte pergunta, apresentada a seguir:

- O tomador de decisão adotaria o contrato futuro?

\subsection{Variáveis analisadas}

As variáveis da abordagem micro analisadas neste trabalho são descritas a seguir.

a)Perfil dos entrevistados

$\rightarrow$ Escolaridade do tomador de decisão

Estudos anteriores como o de Makus et al. (1990), Hanson e Pederson (1998) e Marques (2001), evidenciaram que a maior parte dos usuários de mercados futuros possuem nível superior e segundo grau completo.

Agentes econômicos com baixo nível de escolaridade procuram efetuar estratégias de comercialização com alternativas tradicionais, às quais já estão acostumados, dada a sua falta de compreensão sobre mercados futuros (MARQUES, 2001).

Assim, acredita-se que, quanto maior o nível de escolaridade do tomador de decisão, maior o seu conhecimento sobre mercados futuros e o uso destes.

$\rightarrow$ Idade do tomador de decisão

Quanto menor a idade do tomador de decisão, maior a procura por mercados futuros, pois se acredita que os administradores mais velhos preferem utilizar outros tipos de contratos, em relação ao mercado futuro, pela própria tradição em comercializar a produção nos meios em que já estão habituados (MARQUES, 2001).

Hanson e Pederson (1998) e Marques (2001), por exemplo, demonstraram em seus estudos que gestores com mais de 60 anos utilizam outros tipos de contratos em relação aos contratos futuros.

Assim, na variável perfil dos entrevistados buscouse verificar a idade e o nível de escolaridade dos entrevistados, bem como seu conhecimento de mercados futuros, existência de contatos das empresas com negociação em bolsa e interesse delas em negociar contrato futuro de madeira no Brasil, no sentido de identificar uma demanda potencial por este.

b) Meios de informação

Justifica-se conhecer os meios utilizados para obtenção de informação sobre assuntos relacionados 
com a comercialização de um produto, pois assim se pode concluir sobre a melhor maneira de uma bolsa de comercialização divulgar o mercado futuro desse produto (MARQUES, 2001).

Desse modo, procurou-se identificar os meios utilizados pelos entrevistados para obtenção de informação sobre assuntos referentes à comercialização do insumo florestal, pois isso permite deduzir o canal que deve ser usado pela bolsa para divulgar o mercado futuro da madeira caso ele seja implantado.

\section{c)Renda bruta}

Espera-se que, quanto maior a renda bruta do produtor e do consumidor, maior a probabilidade de procura por mercados futuros, pois, segundo Marques (2001), agentes com elevado nível de renda podem arcar com eventuais custos desses mercados, como depósitos e chamadas de margem. Além disso, maior nível de renda está associado a maiores operações e áreas de plantio e obrigações fixas. Conseqüentemente, necessitam garantir um nível de renda adequado para cobrir as despesas empresariais. Isso faz que eles busquem os mercados futuros.

Assim, apresenta-se, neste tópico, a renda bruta das empresas analisadas no ano de 2004. Essa variável evidencia a situação financeira das empresas e se estas têm recursos suficientes para negociar em bolsa.

\section{d) Financiamento}

Acredita-se que produtores e consumidores que não financiam a produção e o consumo possuem situação financeira melhor. Conseqüentemente, tem maiores condições de operar em mercados futuros (MARQUES, 2001).

Então, buscou-se verificar a existência de financiamento para a produção florestal nas empresas, o porcentual financiado e a parceria utilizada. Isso possibilita dizer se as empresas têm condições financeiras para operar em bolsas, assim como a variável renda bruta.

e)Treinamentos administrativo e econômico

Espera-se daqueles que recebem treinamento administrativo e econômico que compreendam melhor as estratégias de gestão de riscos. Com isso, a probabilidade de usar mercados futuros pode ser maior. São considerados como treinamentos administrativo e econômico a formação superior relacionada a essas áreas e a participação em cursos, palestras etc. (MARQUES, 2001).

Portanto, foi questionado aos entrevistados se os funcionários das empresas recebiam treinamentos econômico e administrativo. Assim, torna-se possível inferir sobre seus conhecimentos de mercados futuros.

f) Riscos da atividade

Espera-se daqueles agentes expostos aos riscos maior procura por proteção contra estes. Assim, acreditase que, quanto maior o risco envolvido numa atividade, maior a busca por estratégias envolvendo mercados futuros.

Nesse sentido, foi questionado aos entrevistados sobre a exposição da empresa ao risco e à sua atitude diante dele. Com isso, é possível concluir se existe possibilidade de busca por proteção em bolsa, através de um contrato futuro de madeira.

\subsection{Fonte de dados}

O instrumento de coleta de dados utilizado foi o questionário composto por questões sobre conhecimento de mercados futuros, escolaridade e idade do tomador de decisão, riscos envolvidos no abastecimento do insumo florestal, meios utilizados para obtenção de informação sobre assuntos relacionados com a comercialização da madeira; negociação em bolsas pelas empresas; financiamento da produção florestal; renda bruta das empresas; atitude diante do risco; e interesse por novas ferramentas de gerenciamento de riscos de preços.

Em $1^{\circ}$ de agosto de 2005, foi realizado um estudo preliminar, ou pré-teste, em uma empresa do setor, com o objetivo de verificar se os termos utilizados nas perguntas eram adequados, bem como identificar a necessidade de incluir novas questões relevantes para a pesquisa ou, mesmo, excluir alguma pergunta julgada desnecessária.

Dez empresas responderam ao questionário no período de 01/08/2005 a 06/10/2005. Em sete empresas, as entrevistas foram realizadas pessoalmente pela pesquisadora, e três empresas responderam ao questionário e o enviaram por e-mail.

Como as questões apresentadas no questionário são de diferentes áreas de conhecimento, alguns

R. Árvore, Viçosa-MG, v.31, n.2, p.307-314, 2007 
entrevistados preferiram repassá-las para outros profissionais da empresa e enviá-las, posteriormente, por e-mail. Todavia, foram excluídas da análise empresas que não produziam celulose com madeira de Eucalyptus. Assim, a amostra foi composta por nove empresas, que em 2003 foram responsáveis por cerca de $82 \%$ da produção nacional de celulose de Eucalyptus.

A amostra compreende, portanto as empresas que são responsáveis pela grande parte do consumo de madeira e também pela grande parte da produção de madeira. Por isso, elas representam bem o mercado de madeira para celulose ou o influenciam muito.

Os dados foram tabulados no Excel e, posteriormente, analisados.

Na Tabela 1 podem ser observadas características das empresas que compõem a amostra e dos entrevistados. Vale ressaltar que os entrevistados são pessoas que exercem certa influência sobre a tomada de decisão da empresa.

\section{RESULTADOS}

\subsection{Abordagem micro}

a) Perfil dos entrevistados

Observa-se, na Tabela 2, que todos os entrevistados possuem curso superior, com predominância em engenharia florestal, a maioria especialização e, uma menor parcela, mestrado.

Como o conhecimento do mercado futuro está relacionado com o nível de escolaridade, foi perguntado aos entrevistados se eles conhecem o mecanismo de mercados futuros. A maioria das respostas foi afirmativa (Tabela 3).
Foi observado que a maioria das empresas de celulose tem experiência com a negociação em bolsa (Tabela 4). As empresas de celulose negociam em bolsa ações e crédito de carbono (Tabela 5). A bolsa mais utilizada para negociação de ações e créditos de carbono é, respectivamente, a BOVESPA e a Chicago Climate Exchange (CCX) (Tabela 6).

Pode-se dizer, assim, que, mesmo alguns entrevistados não tendo conhecimento de mercados futuros, as empresas têm alguma experiência ou estão familiarizadas com negociações em bolsa, fator esse que também pode contribuir para o sucesso de um contrato futuro da madeira no país.

Na Tabela 2, mostra-se, também, que a maior parte dos entrevistados tem entre 25 e 45 anos de idade. Como o uso do mercado futuro está relacionado com a idade, foi perguntado aos entrevistados se haveria interesse, por parte das empresas, em negociar contrato futuro de madeira no Brasil. As respostas evidenciam que, para a maioria das empresas, é interesante adotar contratos futuros (Tabela 7).

Durante as entrevistas, foi possível observar as justificativas para a comercialização de contrato futuro da madeira no Brasil pelas empresas de celulose. Alguns desses depoimentos são explicitados a seguir:

... você tava tendo uma oferta do produto. (...) o sujeito sai de alguma forma catando a laço. Hoje temos vários supervisores atuando regionalizado que, de alguma forma, tão de olho no mercado pra ver onde tem madeira pra comprar. (...) a bolsa seria um local onde a empresa poderia ir diretamente. A empresa saberia onde tem madeira para comprar.

Tabela 1 - Características das empresas e dos entrevistados Table 1 -Characteristic of companies and interviewees

\begin{tabular}{ccccc}
\hline Empresa & Localização & \multicolumn{2}{c}{ Perfil dos Entrevistados } & \multicolumn{2}{c}{ Produção Celulose em 2003 } \\
\cline { 3 - 5 } & & Cormação & Tonelada \\
\hline A & ES & Coord. de fomento & Eng. Florestal & 2.223 .497 \\
B & MG & Coord. de fomento e negócios da madeira & Eng. Florestal & 24,23 \\
C & SP & Ger. de colheita e transporte florestal & Matemática & 885.820 \\
D & RS & Coord. de abastecimento da madeira & Eng. Florestal & 436.856 \\
E & SP & Divisão florestal & Eng. Florestal & 1.391 .216 \\
F & SP & Ger. des. florestal & Eng. Florestal & 130.008 \\
G & SP & Divisão florestal & Eng. Florestal & 1,76 \\
H & SP & Ger. da divisão de recursos naturais & Eng. Florestal & 210.053 \\
I & SP & Diretor florestal & Eng. Florestal & 1.033 .762 \\
\hline
\end{tabular}

Fonte: Resultados da pesquisa e BRACELPA (2004).

R. Árvore, Viçosa-MG, v.31, n.2, p.307-314, 2007 
... devido a incerteza com relação a oferta e preço do insumo...

... pois tem forte comercialização da madeira (...). A empresa já sinalizou com algumas mudanças no portifólio dela e inclui venda desses ativos no Paraná e estamos na eminência de uma futura fábrica de celulose e papel lá. Com isso, a empresa entraria nesse mercado de comercialização, haja vista que este produto ela utilizaria para seu consumo.

... daria garantia de sustentabilidade de matéria-prima para programa de expansão... seria acordado o preço da madeira com bastante antecedência, favorecendo a margem de acerto do planejamento estratégico.

Nota-se, então, que o perfil dos entrevistados é semelhante ao de indivíduos propensos a negociar em bolsa, ou seja, são pessoas relativamente jovens e possuem elevado nível de escolaridade. Além disso, evidenciaram uma provável demanda por contratos futuros de madeira no Brasil, o que pode representar uma vantagem para o seu lançamento no mercado nacional.

Tabela 2 - Perfil dos gestores das empresas de celulose entrevistados na pesquisa

Table 2 - The interviewees' profiles

\begin{tabular}{lc}
\hline Perfil dos Entrevistados & \% de Entrevistados \\
\hline Escolaridade & 100,0 \\
$\quad$ Curso superior & 66,7 \\
Especialização & 22,2 \\
Mestrado & - \\
Doutorado & 88,9 \\
\hline Formação & 11,1 \\
$\quad$ Engenharia florestal & \\
Matemática & - \\
\hline Idade & 66,7 \\
Menos de 25 & 33,3 \\
Entre 25 e 45 & - \\
Entre 46 e 60 & 9 \\
Mais de 60 & \\
\hline Empresas que responderam à questão & \\
\hline
\end{tabular}

Fonte: Resultados da pesquisa.

Tabela 3-Conhecimento dos entrevistados sobre o mecanismo de mercados futuros

Table 3 - The interviewees' knowledge on the mechanism of futures markets

\begin{tabular}{lc}
\hline $\begin{array}{l}\text { Você tem conhecimento } \\
\text { de Mercados Futuros? }\end{array}$ & $\%$ de Entrevistados \\
\hline Sim & 88,9 \\
Não & 11,1 \\
\hline Empresas que responderam à questão & 9 \\
\hline Fonte: Resultados da pesquisa.
\end{tabular}

Tabela 4-Comercialização em bolsas de futuro pelas empresas, segundo os entrevistados

Table 4 -Commercialization in futures exchanges by the companies, according to the interviewees

\begin{tabular}{cc}
\hline A Empresa já Negociou em Bolsa? & \% de Empresas \\
\hline Sim & 77,8 \\
Não & 22,2 \\
\hline Empresas que responderam à questão & 9 \\
\hline
\end{tabular}

Fonte: Resultados da pesquisa.

Tabela 5 - Produtos que as empresas negociam em bolsas de futuro

Table 5 -Products negotiated by the companies in futures exchanges

\begin{tabular}{lc}
\hline Produtos & \% de Empresas \\
\hline Ações & 100,0 \\
Crédito de carbono & 42,9 \\
Commodity & - \\
Taxa de câmbio & - \\
Outros & - \\
\hline Empresas que responderam à questão & 7 \\
\hline
\end{tabular}

Fonte: Resultados da pesquisa.

Tabela 6 - Bolsas de futuro utilizadas pelas empresas para negociações

Table 6 -Futures exchanges used for negotiations by the companies

\begin{tabular}{lc}
\hline Bolsas & \% de Empresas \\
\hline BOVESPA & 85,7 \\
Chicago Climate Exchange (CCX) & 42,9 \\
Bolsa de Nova York & 28,6 \\
Dow Jones & 14,3 \\
BM\&F & - \\
Outras & - \\
\hline Empresas que responderam à questão & 7 \\
\hline
\end{tabular}

Fonte: Resultados da pesquisa.

Tabela 7 - Interesse das empresas em negociar contrato futuro de madeira no Brasil

Table 7 - Interest of companies in negotiating pulpwood futures contract in Brazil

Haveria interesse da Empresa em Negociar Contrato Futuro de Madeira na BM\&F?

\begin{tabular}{cc}
\hline Sim & 55,6 \\
Não & 33,3 \\
Não sabe informar & 11,1 \\
\hline Empresas que responderam à questão & 9 \\
\hline
\end{tabular}

Fonte: Resultados da pesquisa.

R. Árvore, Viçosa-MG, v.31, n.2, p.307-314, 2007 


\section{b) Risco da atividade}

Conforme Tabela 8, a maioria das empresas estão expostas ao risco, isto é, $78 \%$ das empresas.

A incerteza com relação à oferta do insumo florestal é uma fonte de risco para maior parcela de empresas $(77,8 \%)$, seguida por incerteza com relação ao preço $(66,7 \%)$, distância de transporte $(55,6 \%)$ e concorrência pela demanda $(55,6 \%)$ (Tabela 8$)$.

A maioria dos entrevistados colocou a incerteza com relação à oferta do insumo florestal $(71,4 \%)$, em $1^{\circ}$ lugar como principal fonte de risco, seguida pela incerteza com relação ao preço $(33,3 \%)$ e concorrência pela demanda (20\%) (Tabela 8$)$.

Para se protegerem desses riscos, as empresas plantam florestas em áreas próprias, localizadas próximas às fábricas. Assim, observa-se que existe risco envolvido no abastecimento do insumo florestal das empresas e que estas são avessas a eles, o que representa um fator de grande importância para a introdução de um contrato futuro, pois este poderia servir como outro instrumento de proteção. c) Meios de informação

Nota-se, na Tabela 9, que internet e revistas especializadas são os meio de informação utilizados por um número maior de entrevistados $(88,9 \%)$ para obtenção de informações sobre os assuntos relacionados com a comercialização do insumo florestal.

Assim, caso exista um mercado futuro de madeira no Brasil, a bolsa deveria priorizar a sua divulgação na internet e revistas especializadas.

\section{d) Financiamento}

Observa-se, na Tabela 10, que algumas empresas realizam a produção florestal por meio de financiamentos. Para financiar o fomento florestal, a maioria das empresas utiliza recursos do BNDES por meio do PROPFLORA, e a produção própria é financiada por recursos exclusivos do BNDES na maior parte delas, segundo os entrevistados (Tabela 11). Além disso, pode-se verificar, na Tabela 12, que algumas empresas financiam parte da produção florestal, segundo os entrevistados.

Tabela 8 - Fonte de risco envolvida no abastecimento do insumo florestal das empresas

Table 8 -Risk source involved in the forest input supply of the companies

\begin{tabular}{|c|c|c|}
\hline Fontes de Risco e Ordem de Importância & $\%$ de Empresas & $\%$ sobre o Consumo Total \\
\hline Incerteza com relação à oferta da madeira & 77,8 & 73,0 \\
\hline $1^{\circ}$ & 71,4 & \\
\hline $2^{\circ}$ & - & \\
\hline $3^{\circ}$ & 14,3 & \\
\hline $4^{\circ}$ & 14,3 & \\
\hline Incerteza com relação ao preço da madeira & 66,7 & 72,7 \\
\hline $1^{\circ}$ & 33,3 & \\
\hline $2^{\circ}$ & 50,0 & \\
\hline $3^{\circ}$ & 16,7 & \\
\hline $4^{\circ}$ & - & \\
\hline Concorrência na demanda & 55,6 & 72,6 \\
\hline $1^{\circ}$ & 20,0 & \\
\hline $2^{\circ}$ & 60,0 & \\
\hline $3^{\circ}$ & 20,0 & \\
\hline $4^{\circ}$ & 20,0 & \\
\hline Distância de transporte & 55,6 & 72,8 \\
\hline $1^{\circ}$ & - & \\
\hline $2^{\circ}$ & 20,0 & \\
\hline $3^{\circ}$ & 40,0 & \\
\hline $4^{\circ}$ & 40,0 & \\
\hline \multicolumn{3}{|l|}{ Outros tipos de riscos } \\
\hline Legislação rigorosa & 11,1 & \\
\hline Não existem riscos & 22,2 & 27,0 \\
\hline Empresas que responderam à questão & 9 & $20.866 .349 \mathrm{~m}^{3}$ \\
\hline
\end{tabular}

Fonte: Resultados da pesquisa. 
Tabela 9-Meios utilizados pelos entrevistados para obtenção de informação sobre os assuntos relacionados com a comercialização do insumo florestal

Table 9 -Approaches used by the interviewees to obtain information on the issues related with the forest input commercialization

\begin{tabular}{lc}
\hline Meios de Informação & \% de Entrevistados \\
\hline Revistas especializadas & 88,9 \\
Internet & 88,9 \\
Jornais & 55,6 \\
TV & 55,6 \\
Outros & 66,7 \\
$\quad$ Bentchmarck & 83,3 \\
Consultorias & 16,7 \\
$\quad$ Monitoramento & 16,7 \\
Clientes & 16,7 \\
$\quad$ Fornecedores & 16,7 \\
\hline \multicolumn{2}{c}{ Empresas que responderam à questão } \\
\hline \multirow{2}{*}{ Fonte: Resultados da pesquisa. }
\end{tabular}

Fonte: Resultados da pesquisa.

Pode-se pensar, assim, que as empresas que financiam a produção florestal não possuem condições financeiras suficientes para operar em bolsa. Porém, o financiamento da produção florestal ocorre porque o capital oferecido pelo governo é mais barato, como pode ser observado no depoimento a seguir.

... a empresa financia toda a produção florestal porque o capital oferecido pelo governo é mais barato (...), e não porque a empresa não tem recurso para isso....
Tabela 10 - Financiamento da produção florestal pelas empresas Table 10 - Financing of forest production by the companies

\begin{tabular}{lc}
\hline A Empresa Financia: & \% de Empresas \\
\hline o fomento florestal? & 55,6 \\
Sim & 22,2 \\
Não & 22,2 \\
Não possui fomento florestal & 44,4 \\
\hline a produção própria? & 55,6 \\
$\quad$ Sim & 9 \\
\hline Não
\end{tabular}

Fonte: Resultados da pesquisa.

Assim, o fato de algumas empresas financiarem a produção florestal não significa, necessariamente, excassez de recursos. Acredita-se, então, que isso não prejudique o desenvolvimento de um contrato futuro de madeira no país.

\section{e) Renda bruta}

Conforme Tabela 13, as empresas possuem elevada renda bruta, segundo os entrevistados. Isso indica que elas têm condições financeiras suficientes para arcar com os custos de uma negociação a futuro em bolsa.

\section{d) Treinamento}

Os dados coletados indicam que, em grande parte das empresas, os funcionários recebem treinamentos econômicos e administrativo (Tabela 14).

Tabela 11 - Parceria utilizada pelas empresas para financiar a produção florestal Table 11 - Partnership used by the companies to finance forest production

\begin{tabular}{|c|c|c|c|c|c|c|}
\hline \multirow[t]{2}{*}{ Produção Florestal } & \multirow{2}{*}{$\begin{array}{c}\text { Empresas que } \\
\text { Responderam à Questão }\end{array}$} & \multicolumn{5}{|c|}{ Parceria } \\
\hline & & BNDES & PRONAF & FINAME & PROPFLORA & Outros \\
\hline & & \multicolumn{5}{|c|}{$\%$ de empresas } \\
\hline Fomento florestal & 5 & 60,0 & 20,0 & - & 40,0 & 20,0 \\
\hline Produção própria & 4 & 75,0 & - & 25,0 & - & 25,0 \\
\hline
\end{tabular}

Fonte: Resultados da pesquisa.

Tabela 12 - Parcela da produção florestal que as empresas financiam Table 12 - Part of the forest production financed by the companies

\begin{tabular}{|c|c|c|c|c|c|c|}
\hline \multirow[t]{2}{*}{ Produção Florestal } & \multirow{2}{*}{$\begin{array}{c}\text { Empresas que } \\
\text { Responderam à Questão }\end{array}$} & \multicolumn{5}{|c|}{$\%$ da Produção Florestal Financiada } \\
\hline & & $100 \%$ & $70 \%$ a $99 \%$ & $50 \%$ a $69 \%$ & $30 \%$ a $49 \%$ & Menos de $30 \%$ \\
\hline & & \multicolumn{5}{|c|}{$\%$ de empresas } \\
\hline Fomento florestal & 5 & 40,0 & - & 20,0 & 20,0 & 20,0 \\
\hline Produção própria & 4 & 50,0 & - & 50,0 & - & - \\
\hline
\end{tabular}

Fonte: Resultados da pesquisa. 
Tabela 13 - Renda bruta das empresas, em 2004 Table 13 - Companies income, in 2004

\begin{tabular}{lc}
\hline Renda Bruta (R $\$$ milhões) & \% de Empresas \\
\hline Mais de 3.000 .000 & 85,7 \\
1.000 .001 a 3.000 .000 & 14,3 \\
600.000 a 1.000 .000 & - \\
\hline Empresas que responderam à questão & 7 \\
\hline
\end{tabular}

Fonte: Resultados da pesquisa.

Tabela 14 - Parcela de empresas que possuem funcionários que recebem treinamento na área de economia e administração

Table 14 - Amount of companies having employees being trained in economics and administration

\begin{tabular}{lc}
\hline Os Funcionários da Empresa & \% de Empresas \\
Recebem Treinamento & \\
Econômico e Administrativo? & 88,9 \\
\hline \multicolumn{1}{c}{ Sim } & 11,1 \\
\hline Não & 9 \\
\hline
\end{tabular}

Fonte: Resultados da pesquisa.

Acredita-se, então, que esses funcionários tenham algum conhecimento de mercados futuros, o que, segundo a teoria, pode contribuir para o sucesso de um contrato futuro de madeira no país.

\section{CONCLUSÕES}

Do presente trabalho, é possível concluir, considerando apenas a abordagem micro de Pennings e Leuthold (1999), que é viável a implantação de um mercado de contrato futuro da madeira de reflorestamento de Eucalyptus spp para produção de celulose no país. Os fatores determinantes para isso são:

- Aqueles que influenciam o processo de decisão nas empresas de celulose possuem elevado nível de escolaridade, e a maioria conhece o processo de negociação em bolsa. Além disso, são pessoas relativamente jovens e evidenciam uma provável demanda por contrato futuro de madeira pelas empresas nacionais.

- As empresas de celulose no país já estão familiarizadas com a negociação em bolsas.
- Existe risco envolvido na atividade florestal da maioria das empresas, relacionado à oferta do insumo florestal, à concorrência, à distância de transporte e ao preço. As empresas poderiam adotar o contrato futuro como instrumento de gestão de riscos.

- Todas as empresas, sob análise, têm condições financeiras para operar em bolsas, e na grande maioria os funcionários recebem treinamentos econômico e administrativo.

\section{AGRADECIMENTOS}

Ao Conselho Nacional de Desenvolvimento Científico e Tecnológico (CNPq), pela concessão da bolsa de estudo; ao professor Danilo Rolim Dias de Aguiar, pela sugestão metodológica; e aos entrevistados, pelas informações.

\section{6 - REFERÊNCIAS}

HANSON, D.K.; PEDERSON, G. Price risk management by Minnesota farmers. Agricultural Economist, n. 691, winter 1998.

MAKUS, L. D. et al. Factors influencing farm level use of futures and options in commodity marketing. Agribusiness, v.6, n.6, p.621-631, 1990.

MARQUES, R.H.S. Determinantes do uso de mercados futuros pelos produtores de soja de Cascavel - PR. 2001. $110 \mathrm{f}$. Dissertação (Mestrado em Economia Rural) Universidade Federal de Viçosa, Viçosa, 2001.

PENNINGS, J.M.E.; LEUTHOLD, R. M.

Commodity futures contract viability: a multidisciplinary approach. Illinois: 1999. Disponível em: < http://www.ace.uiuc. edu/ ofor/ofor9902.pdf>. Acesso em: 08/05/2004.

OLIVEIRA, P.R.; VALVERDE, S.R.; COELHO, F.M.G. Aspectos de relevância econômica no fomento florestal a partir da percepção dos produtores rurais envolvidos. Revista Arvore, v.30, n.4, p.593-602, 2006. 